\title{
Working memory networks and activation patterns in schizophrenia and bipolar disorder: comparison with healthy controls
}

Christine Lycke Brandt, Tom Eichele, Ingrid Melle, Kjetil Sundet, Andrés Server, Ingrid Agartz, Kenneth Hugdahl, Jimmy Jensen and Ole A. Andreassen

\section{Background}

Schizophrenia and bipolar disorder are severe mental

disorders with overlapping genetic and clinical

characteristics, including cognitive impairments. An important question is whether these disorders also have overlapping neuronal deficits.

\section{Aims}

To determine whether large-scale brain networks associated with working memory, as measured with functional magnetic resonance imaging (fMRI), are the same in both schizophrenia and bipolar disorder, and how they differ from those in healthy individuals.

\section{Method}

Patients with schizophrenia $(n=100)$ and bipolar disorder $(n=100)$ and a healthy control group $(n=100)$ performed a 2-back working memory task while fMRI data were acquired. The imaging data were analysed using independent component analysis to extract large-scale networks of task-related activations.

\section{Results}

Similar working memory networks were activated in all groups. However, in three out of nine networks related to the experimental task there was a graded response difference in fMRI signal amplitudes, where patients with schizophrenia showed greater activation than those with bipolar disorder, who in turn showed more activation than healthy controls. Secondary analysis of the patient groups showed that these activation patterns were associated with history of psychosis and current elevated mood in bipolar disorder.

\section{Conclusions}

The same brain networks were related to working memory in schizophrenia, bipolar disorder and controls. However, some key networks showed a graded hyperactivation in the two patient groups, in line with a continuum of neuronal abnormalities across psychotic disorders.

\section{Declaration of interest}

K.H. has stock ownership in NordicNeuroLab, which supplied audiovisual and other hardware equipment for the fMRI image acquisitions as well as software for fMRI data analysis. I.A. has served as an unpaid consultant for Eli Lilly. I.M. has received speaker's honoraria from Janssen and AstraZeneca. O.A.A. has received speaker's honoraria from AstraZeneca, Janssen, Bristol-Myers Squibb and GlaxoSmithKline.
Schizophrenia and bipolar disorder have similar clinical symptoms, and overlapping genetic factors have been implicated. ${ }^{1-4}$ Further, impairment of cognitive functions such as working memory is seen in both disorders. ${ }^{5,6}$ However, a limited number of studies have examined whether these disorders also have overlapping neural substrates related to cognitive task performance, ${ }^{7-10}$ as measured for example with functional magnetic resonance imaging (fMRI). Impairment of working memory is considered a core feature of schizophrenia. ${ }^{5}$ Although deficits are present also in bipolar disorder, this has received less attention than in schizophrenia. In both disorders the impairment is stable over time and across different clinical states, is relatively resistant to treatment and contributes to poor functional outcome. ${ }^{5}$ In addition, neuroimaging studies have shown altered working memory activation in both disorders, ${ }^{11,12}$ but only two previous studies of working memory have compared schizophrenia and bipolar disorder in the same experiment, ${ }^{13,14}$ which makes working memory an important study target. Since it is a trait in both disorders, neural activation measured with fMRI during a working memory task can determine overlapping neurobiological substrates. Working memory involves not only the dorsolateral prefrontal cortex but also distributed brain networks, ${ }^{15,16}$ and may therefore reflect more widely distributed abnormalities that could be present in both disorders. ${ }^{12,13}$ Although the working memory activation pattern has been well characterised in schizophrenia, ${ }^{12}$ it is less clear in bipolar disorder. Further, it remains unclear whether the working memory activation patterns differ between the disorders with regard to brain regions or activation levels (indicating differences in mechanisms). To draw conclusions about overlapping neurobiological substrates of working memory in schizophrenia and bipolar disorder, the groups should be compared in the same experiment, also including a healthy control group. Owing to the heterogeneity of patient samples, large well-characterised samples are needed. ${ }^{12,17}$ We used fMRI and independent component analysis (ICA) to investigate brain networks involved in working memory in people with schizophrenia $(n=100)$, people with bipolar disorder $(n=100)$ and healthy volunteers $(n=100)$. Since the disorders share genetic susceptibility and have similar neurocognitive manifestations, $1,2,4,5,18$ we predicted that the two patient groups would have impairments in the same brain networks, and that there would be differences in both working memory performance and activation levels of the underlying networks in patients compared with controls. In addition, we explored how clinical, cognitive and behavioural factors were associated with the activation patterns across diagnostic groups.

\section{Method}

In total, 300 participants were included in the study, of whom 100 had a DSM-IV diagnosis of schizophrenia spectrum disorder (schizophrenia $n=75$, schizoaffective disorder $n=14$, 
schizophreniform disorder $n=11$ ) and 100 had a diagnosis of bipolar disorder (type $1 n=57$, type $2 n=43$ ). ${ }^{19}$ These groups are subsequently referred to as 'schizophrenia' and 'bipolar disorder' respectively. In addition, 100 healthy control participants were included. Patients were recruited consecutively from psychiatric units at four major hospitals in Oslo. The control group was randomly selected from the same catchment area as the patient group using statistical records (www.ssb.no). Of the control group, $28 \%$ replied to the invitation and consented to participate, and of these $37 \%$ underwent scanning. The current study is part of the Thematically Organised Psychosis (TOP) study, ${ }^{6}$ and is approved by the Regional Committee for Medical Research Ethics and the Norwegian Data Inspectorate. After complete description of the study to the participants, written informed consent was obtained according to the Declaration of Helsinki. To participate in the study all individuals had to be aged 18-65 years, speak a Scandinavian language and have the ability to undergo fMRI examination. Exclusion criteria were presence of a developmental disorder or serious brain damage, or having a metal implant or cardiac pacemaker. To ensure a healthy control group, these participants were screened with a questionnaire about severe mental illness and the Primary Care Evaluation of Mental Disorders, ${ }^{20}$ and were excluded if they or any of their first-degree relatives had a lifetime history of a severe psychiatric disorder (schizophrenia, bipolar disorder or major depression).

\section{Clinical assessment}

Patients were clinically characterised through a personal interview conducted by trained clinicians working as research fellows (MDs or psychologists). The interview covered diagnostics, symptoms, neurocognition, drug use and medication status. ${ }^{6}$ The psychiatric diagnosis was established using the Structured Clinical Interview for DSM-IV Axis I Disorders (SCID-I). ${ }^{21}$ Diagnostic reliability was satisfactory, ${ }^{22}$ with overall agreement for DSM-IV diagnosis categories of $82 \%$ and overall $\kappa=0.77$ (95\% CI $0.60-0.94)$. Information from the SCID assessment showed that $56 \%$ of the patients were in remission from either affective or psychotic episode (bipolar disorder 74\%, schizophrenia 37\%). Of those not in remission (44\%), $30 \%$ of the total number of patients were experiencing a psychotic episode (bipolar disorder 1\%, schizophrenia $60 \%$ ) and $14 \%$ an affective episode (bipolar disorder: $3 \%$ mania, $1 \%$ hypomania, $21 \%$ depression; schizophrenia: $3 \%$ depression). Complete data were missing for $17 \%$ of the patients. Symptom assessment on the day of scanning showed that psychotic or affective symptoms were present in less than a third of the patients in either diagnostic group, indicating that most patients had low symptom levels during the experimental task. The scanning was done on a different day from the diagnostic interview, so current symptom levels were assessed also on the day of scanning, using an interview covering symptoms of psychosis, depression and elevated mood in the previous week. ${ }^{23}$ The symptoms were rated as present, possibly present or absent. In addition, regular psychopharmacological medication was recorded, as well as the use of alcohol and non-alcohol drugs (see Table 1).

\section{Experimental task}

The working memory task was an $n$-back task involving consecutive presentations of pairs of numbers between 1 and $9 .{ }^{24,25}$ Since the aim of the study was to investigate brain networks, we applied only a 2-back condition, in which the participant was asked to press a response button when the two numbers were the same as the numbers shown two trials back in the sequence. The stimulus presentations were implemented in E-Prime software (version 1.0; Psychology Software Tools, Sharpsburg, Pennsylvania, USA) and were presented through video goggles using the VisualSystem (NordicNeuroLab, Bergen, Norway). Behavioural responses were collected using the ResponseGrips system (NordicNeuroLab, Bergen, Norway) and the participants responded with their right or left index finger or thumb. Before scanning all participants underwent a short training procedure to ensure that they understood the task instructions. Immediately before the start of the experiment, task instructions were also shown in the goggles. The task was presented using a block design with four ON blocks (stimuli) and four OFF blocks (no stimulus). In each ON block there were 18 stimulus presentations, with 3 or 4 presentations being pseudo-randomised target stimuli (in total 13 targets). The stimulus duration was $300 \mathrm{~ms}$ and the interstimulus interval was $2500 \mathrm{~ms}$. An ON block lasted for $52 \mathrm{~s}$ and was followed by an OFF block of $26 \mathrm{~s}$ during which only a fixation cross was presented and no task was performed.

\section{Image acquisition}

The data were acquired on a $1.5 \mathrm{~T}$ Siemens Magnetom Sonata (Siemens Medical Solutions, Erlangen, Germany) supplied with a standard head coil at Oslo University Hospital, Ullevål. Initial scanning of anatomy was done with a magnetisation prepared rapid acquisition gradient echo (MPRAGE) $T_{1}$-weighted sequence with 80 sagittal slices: repetition time (TR) $2000 \mathrm{~ms}$, echo time (TE) $3.95 \mathrm{~ms}$, field of view (FOV) $256 \times 256 \mathrm{~mm}^{2}$, flip angle (FA) $7^{\circ}$, matrix $128 \times 128$. Next, $T_{2}{ }^{*}$-weighted functional imaging with 164 blood oxygen level-dependent (BOLD) whole brain measurements per condition was done with an echoplanar imaging (EPI) pulse sequence. Each EPI volume measurement consisted of 24 axial slices $(\mathrm{TR}=2040 \mathrm{~ms}, \mathrm{TE}=50 \mathrm{~ms}$, FOV $224 \times 224 \mathrm{~mm}^{2}, \mathrm{FA}=90^{\circ}$, matrix $64 \times 64$ ), with a pixel size of $3 \mathrm{~mm}$ in the axial plane and a slice thickness of $4 \mathrm{~mm}$ with $1 \mathrm{~mm}$ gap between slices. The first seven volumes and the last volume were discarded to avoid initial steady-state effects, leaving 156 images for analysis.

\section{Preprocessing}

Preprocessing of the fMRI data was done using the SPM5 software package (Wellcome Department of Imaging Neuroscience, www. fil.ion.ucl.ac.uk/spm/software/spm5/) implemented in Matlab 7.1 (MathWorks, Natick, Massachusetts, USA) running on a Windows platform. The preprocessing included realignment, spatial normalisation and smoothing, using a $6 \mathrm{~mm}$ full-width half-maximum smoothing kernel.

\section{Independent component analysis}

Group ICA was performed with the Group ICA of fMRI Toolbox (GIFT) (http://icatb.sourceforge. net/). First, the preprocessed fMRI data from each participant were intensity normalised through voxel-wise division of the time series mean (equivalent to percentage signal change) and global intensity offsets were removed between individual data-sets. Then, principal component analysis (PCA) was used to reduce the data from each participant. For the analyses presented here the number of retained principal components was set to 36 , since this model order was the maximum across dimensionality estimations for each participant and yielded robust Icasso results. ${ }^{26}$ Using the Infomax ICA algorithm, ${ }^{27}$ group sources in the aggregate data were computed. To reduce sensitivity to initial conditions of Infomax and thereby estimate robust components, the ICA decomposition was repeated 100 times using the Icasso algorithm, ${ }^{26}$ and back-reconstruction 
(done with GICA3) was based on the centrotypes of component clusters. The resulting 36 independent components from the decomposition, with group average spatial maps and time courses, were inspected to identify and discard those that were physiological components of no interest, such as those located primarily in the ventricles, large venous and arterial vessels and white matter, as well as edge effects due to motion $(n=10)$. Components that did not show clear and significant box-car type activation patterns in the average time courses and $t$-tests of the mean difference between ON blocks and OFF blocks $(n=17)$ were also discarded. The remaining 9 components ( 6 activations and 3 deactivations) were used in further analyses reported here.

\section{Statistical analysis}

The amplitude data from the component time courses were submitted to an analysis of covariance (ANCOVA) model with gender and age as covariates to test for main effects of group, and post hoc $t$-tests were used to investigate differences between groups (control $v$. bipolar disorder, bipolar disorder $v$. schizophrenia and schizophrenia $v$. control). The same analysis was conducted for the spatial maps in order to detect topographical features that supported a group difference after correction for covariates. Gender and age were included as covariates in all analyses, since these variables were significantly different between groups, but not directly linked to the illness.

\section{Secondary analyses}

For the independent components that showed group differences in the ANCOVA, follow-up analyses were conducted to investigate the effects of factors that might explain the main results, after correcting for gender and age. Individuals with missing data on the specific variables were excluded from the analysis including that variable.

Cognitive, demographic and substance use factors. For the total sample of 300 individuals, linear regression with group as a fixed factor was performed to test the effect of handedness, education, IQ score, alcohol use, non-alcohol drug use and smoking separately on the amplitude values, and to investigate to what degree each variable influenced the group effect from the main analysis.

Clinical variables. Linear regression was performed in the two patient groups separately to test the effect of lifetime episodes (history of psychosis, depression or mania), current symptoms (psychotic, depressive or elevated mood), illness duration and medication (antipsychotics, anti-epileptics/lithium, antidepressants, anxiolytics) separately on the amplitude values. Regarding lifetime psychotic or mood episodes, we focused on psychosis in the bipolar disorder group and depression and mania in the schizophrenia group, because the presence of psychotic episodes varies most in bipolar disorder (included in the diagnostic criteria of schizophrenia) and the variation in mood episodes is largest in schizophrenia (included in the diagnostic criteria of bipolar disorder). For the bipolar disorder group the effect of subgroup (type 1 or 2) was also tested. Similarly, the effect of schizophrenia spectrum subgroup (schizoaffective disorder, schizophreniform disorder or schizophrenia) was tested in the schizophrenia group.

Behavioural variables. For the total sample, linear regression with group as a fixed factor was performed to test the effects of accuracy and response time separately on the amplitude values, and to investigate to what degree each variable influenced the group effect from the main analysis. Further, the effects of accuracy and response time on the amplitude values were tested in each group separately.

\section{Results}

The demographic and clinical characteristics of the study participants are shown in Table 1 (a fully annotated version is given in online Table DS1).

\section{Behavioural data}

Performance on the working memory task, corrected for gender and age, was significantly different between the groups, with respect to both response accuracy $(F=18.3, P<0.001)$ and response time $(F=9.6, P<0.001)$ (Table 2$)$. Both task performance measures showed a graded pattern, with results for participants with bipolar disorder lying between those of the control group and the schizophrenia group, with the control group showing the highest accuracy and the shortest response time (Fig. 1). For response accuracy there were significant differences for all group comparisons: schizophrenia $v$. bipolar disorder $(P<0.01)$, bipolar disorder $v$. controls $(P<0.01)$ and schizophrenia $v$. controls $(P<0.001)$. For response time there were significant differences between the patient groups $(P<0.05)$ and for schizophrenia $v$. controls $(P<0.001)$. Patients with bipolar disorder were not significantly slower than controls $(P=0.22)$.

Similarly to the procedure for the fMRI data, we tested whether behavioural differences between groups could be explained by any of the demographic or clinical variables. First, ANCOVAs for the whole sample were performed to test the effect of handedness, education, IQ score, alcohol use and non-alcohol drug use separately on the behavioural data, corrected for gender and age. Then, ANCOVAs for each patient group were performed for the clinical variables (lifetime episodes, current symptoms level, illness duration and medication). The results for the whole sample showed that response accuracy was moderately dependent on education $(t=4.0, P<0.001)$ and IQ score $(t=7.2, P<0.001)$, and that response time marginally decreased with alcohol use $(t=2.3, P<0.05)$. In the bipolar disorder group, response time was slightly shorter for patients with a history of depression $(t=2.1, P<0.05)$ and current symptoms of depression $(t=2.1$, $P<0.05)$, and longer for patients with a history of mania $(t=3.2, \quad P<0.05)$. Response time also increased with illness duration $(t=2.1, \quad P<0.05)$ in the bipolar disorder group. Individuals with missing data on any of the variables were excluded from the particular analyses.

\section{Imaging data}

Brain regions involved in the nine independent components that showed the largest signal changes in the working memory task across all participant groups are shown in Table 3 and Fig. 2 (left panel). Components 1-6 represent activations and components 7-9 represent deactivations that were correlated with the task. Figure 2 (right panel) also shows bar plots for the amplitude differences between groups for all components when variance accounted for by gender and age is removed from the data. Components 1, 3 and 4 showed significant ANCOVA differences, with a graded response pattern whereby patients in the schizophrenia group showed greater activation than those in the bipolar disorder group, who in turn showed more activation than the control group. The post hoc $t$-tests yielded significant differences between control and schizophrenia group participants in all three components, and between bipolar disorder and schizophrenia groups in component 1 (Table 4). Component 1 consisted of 


\begin{tabular}{|c|c|c|c|c|c|c|}
\hline & \multirow{2}{*}{$\begin{array}{l}\text { Schizophrenia group } \\
\qquad(n=100)\end{array}$} & \multirow{2}{*}{$\begin{array}{l}\text { Bipolar disorder group } \\
\qquad(n=100)\end{array}$} & \multirow{2}{*}{$\begin{array}{l}\text { Control group } \\
\qquad(n=100)\end{array}$} & \multicolumn{3}{|c|}{ Analysis } \\
\hline & & & & Test & $P$ & Post hoc \\
\hline \multicolumn{7}{|l|}{ Demographics } \\
\hline Gender (male), $n$ (\%) & $67(67)$ & $40(40)$ & $50(50)$ & $\chi^{2}=14.9$ & 0.001 & $\mathrm{BD}, \mathrm{HC}<\mathrm{SZ}$ \\
\hline Age, years: mean (s.d.) & $31.9(7.7)$ & $35.4(11.4)$ & $34.8(9.1)$ & $F=3.9$ & 0.021 & $\mathrm{SZ}<\mathrm{BD}$ \\
\hline Handedness (right), $n$ (\%) & $87(87)$ & $89(89)$ & $93(93)$ & $\chi^{2}=2.0$ & 0.365 & \\
\hline Education, years: mean (s.d) & $13.0(2.4)$ & $13.3(2.3)$ & $14.4(2.3)$ & $F=10.4$ & $<0.001$ & $\mathrm{SZ}, \mathrm{BD}<\mathrm{HC}$ \\
\hline IQ score: mean (s.d.) & $104.7(14.8)$ & $108.1(12.5)$ & $114.8(10.4)$ & $F=16.2$ & $<0.001$ & $\mathrm{SZ}, \mathrm{BD}<\mathrm{HC}$ \\
\hline Duration of illness, years: mean (s.d.) & $6.4(6.2)$ & $6.7(7.2)$ & & $t=0.3$ & 0.736 & \\
\hline \multicolumn{7}{|l|}{ Comorbid disorders, $n(\%)$} \\
\hline Substance use & $23(23)$ & $23(23)$ & & $\chi^{2}=0.0$ & 1.00 & \\
\hline Somatic illness & $10(11)$ & $12(13)$ & & $\chi^{2}=0.29$ & 0.592 & \\
\hline \multicolumn{7}{|l|}{ Lifetime episodes, $n$ (\%) } \\
\hline Psychosis & $100(100)$ & $52(52)$ & & $\chi^{2}=63.2$ & $<0.001$ & \\
\hline Psychosis in bipolar disorder types $1 / 2$ & & $45(79) / 7(16)$ & & $\chi^{2}=38.6$ & $<0.001$ & \\
\hline Depression & $56(56)$ & $91(91)$ & & $\chi^{2}=31.5$ & $<0.001$ & \\
\hline Mania & $8(8)$ & $57(57)$ & & $\chi^{2}=54.7$ & $<0.001$ & \\
\hline \multicolumn{7}{|l|}{ Current symptoms, $n$ (\%) } \\
\hline Psychotic symptoms & $29(30)$ & $17(17)$ & & $\chi^{2}=4.1$ & 0.043 & \\
\hline Elevated mood symptoms & $8(8)$ & $14(14)$ & & $\chi^{2}=1.8$ & 0.175 & \\
\hline Depressive symptoms & $24(24)$ & $31(32)$ & & $\chi^{2}=1.2$ & 0.266 & \\
\hline \multicolumn{7}{|l|}{ Medication } \\
\hline \multicolumn{7}{|l|}{ Antipsychotics } \\
\hline$n(\%)$ & $71(76)$ & $35(38)$ & & $\chi^{2}=27.0$ & $<0.001$ & \\
\hline DDD, mean (s.d.) & $1.10(1.15)$ & $0.29(0.52)$ & & $t=6.2$ & $<0.001$ & \\
\hline \multicolumn{7}{|l|}{ Anti-epileptics/lithium } \\
\hline$n(\%)$ & $8(8)$ & $58(64)$ & & $\chi^{2}=63.2$ & $<0.001$ & \\
\hline DDD, mean (s.d.) & $0.06(0.30)$ & $0.57(0.63)$ & & $t=7.1$ & $<0.001$ & \\
\hline \multicolumn{7}{|l|}{ Antidepressants } \\
\hline$n(\%)$ & $23(25)$ & $26(29)$ & & $\chi^{2}=0.4$ & 0.556 & \\
\hline DDD, mean (s.d.) & $0.35(0.73)$ & $0.42(0.80)$ & & $t=0.6$ & 0.553 & \\
\hline \multicolumn{7}{|l|}{ Anxiolytics } \\
\hline$n(\%)$ & $7(8)$ & $7(8)$ & & $\chi^{2}=0.0$ & 1.00 & \\
\hline DDD, mean (s.d.) & $0.06(0.21)$ & $0.10(0.37)$ & & $t=0.9$ & 0.396 & \\
\hline \multicolumn{7}{|l|}{ Substance use } \\
\hline Alcohol use (AUDIT score), mean (s.d.) & $5.94(6.13)$ & $7.10(6.02)$ & $5.24(3.25)$ & $F=3.09$ & 0.047 & $\mathrm{HC}<\mathrm{BD}$ \\
\hline $\begin{array}{l}\text { Non-alcohol drug use (DUDIT score), } \\
\text { mean (s.d.) }\end{array}$ & 2.53 (5.99) & $2.26(6.35)$ & $0.29(1.65)$ & $F=5.49$ & 0.005 & $\mathrm{HC}<\mathrm{BD}, \mathrm{SZ}$ \\
\hline Smoking, $n(\%)$ & $37(49)$ & $39(50)$ & NA & $\chi^{2}=0.01$ & 0.934 & \\
\hline
\end{tabular}

\begin{tabular}{|c|c|c|c|c|c|c|}
\hline & \multirow{2}{*}{$\begin{array}{l}\text { Schizophrenia group } \\
\qquad(n=100)\end{array}$} & \multirow{2}{*}{$\begin{array}{l}\text { Bipolar disorder group } \\
\qquad(n=100)\end{array}$} & \multirow{2}{*}{$\begin{array}{l}\text { Control group } \\
\quad(n=100)\end{array}$} & \multicolumn{3}{|c|}{ ANCOVA } \\
\hline & & & & $F$ & P & Post hoc \\
\hline Accuracy, mean (s.e.) & $0.936(0.01)$ & $0.958(0.01)$ & $0.979(0.01)$ & 18.3 & $<0.001$ & $\mathrm{SZ}<\mathrm{BD}<\mathrm{HC}$ \\
\hline Response time, hits, ms: mean (s.e. $)^{a, b, c}$ & $690(19.3)$ & $615(18.7)$ & $575(17.8)$ & 9.6 & $<0.001$ & $\mathrm{SZ}>\mathrm{HC}, \mathrm{SZ}>\mathrm{BD}$ \\
\hline
\end{tabular}

frontal and parietal brain regions (overlapping with a frontoparietal network found to be involved in, for example, working memory), component 3 involved superior/inferior parietal regions (overlapping with a part of the dorsal attentional network) and component 4 included (for example) the insula and inferior frontal gyri (overlapping with a part of the executive control/salience network). ${ }^{28}$ For the remaining six components there was no significant group difference in the ANCOVA. However, there was a graded response similar to the one described above (controls $<$ bipolar disorder $<$ schizophrenia) also for component 6 , whereas components 5 and 9 showed an inverse gradation (controls $>$ bipolar disorder $>$ schizophrenia). When comparing the spatial maps of these nine components between groups, no considerable differences were found within the activation maxima of the particular components. However, in component 8 there was a significantly stronger activation in schizophrenia compared with bipolar disorder in an area of the right superior temporal gyrus (Montreal Neurological Institute peak coordinates 39, 12, -18 ; $t=5.9, P<0.001$, family-wise error-corrected).

\section{Secondary analyses}

Exploratory post hoc analyses aimed to investigate the main results more deeply, to find out if and how different variables could 


\begin{tabular}{|c|c|c|c|}
\hline & Hemisphere & Brain regions ${ }^{a}$ & Peak voxel ${ }^{b}$ \\
\hline \multicolumn{4}{|l|}{ Activations } \\
\hline \multirow[t]{5}{*}{ Component 1} & $\mathrm{R}$ & MFG (46) & \\
\hline & L & MFG (9) & \\
\hline & $L+R$ & Ins (48) & \\
\hline & $L+R$ & PreCG (6), SMA (6, 32), MFG (6), SFG (6), MCC $(6,32)$ & $-6,6,57$ \\
\hline & $L+R$ & IPL (40), SPL (7) & \\
\hline \multirow[t]{4}{*}{ Component 2} & $\mathrm{R}$ & MFG $(6,8,9,44,45,46)$, SFG (10), IFG (47), mSFG (8) & \\
\hline & $\mathrm{L}$ & MFG (10) & \\
\hline & $L+R$ & IPL (40), AG (7, 39) & $45,-57,48$ \\
\hline & $\mathrm{R}$ & $\operatorname{Prec}(7)$ & \\
\hline \multirow[t]{2}{*}{ Component 3} & $L+R$ & PostCG (2), IPL (40), AG (7), SPL (7), SMG (7) & $-42,-36,45$ \\
\hline & $L+R$ & ITG (37) & \\
\hline \multirow[t]{3}{*}{ Component 4} & $L+R$ & IFG $(45,47,48)$, Ins (48), R PreCG (6) & $-42,18,-6$ \\
\hline & $L+R$ & SMA (6) & \\
\hline & $L+R$ & SMG $(2,40,48)$, IPL (40), STG (42), R MTG $(21,22)$ & \\
\hline \multirow[t]{4}{*}{ Component 5} & L & IFG $(44,45,47,48)$, MFG (44/46/6/8), PreCG (6) & \\
\hline & L & SMA (8) & \\
\hline & L & IPL (40/39), SPL (7), AG (39) & $-36,-66,45$ \\
\hline & L & $\operatorname{Prec}(7)$ & \\
\hline Component 6 & $L+R$ & Prec (5/7), SPL (7) & $-6,-63,57$ \\
\hline \multicolumn{4}{|l|}{ Deactivations } \\
\hline \multirow{3}{*}{ Component 7} & $L+R$ & SFG (8), SMA (8) & \\
\hline & $L+R$ & PreCG (6), PostCG (2/3/4), SMA (6), SPL (2), Prec, MCC (23) & $33,-36,63$ \\
\hline & $L+R$ & RO (48) & \\
\hline \multirow[t]{4}{*}{ Component 8} & $L+R$ & Prec (23/30), CS (23) & \\
\hline & $L+R$ & FFG (30/37) & \\
\hline & $L+R$ & MTG (39), MOG (39/19), AG (39/19) & $48,-75,30$ \\
\hline & $L+R$ & SMG $(2 / 40)$ & \\
\hline \multirow[t]{3}{*}{ Component 9} & $L+R$ & mSFG (9/10/32), ACC (32), SFG (9) & $0,48,-3$ \\
\hline & $L+R$ & STP (38) & \\
\hline & $L+R$ & Prec (23), PCC (23) & \\
\hline
\end{tabular}

\begin{tabular}{|c|c|c|c|c|c|c|}
\hline \multirow[b]{2}{*}{ Component } & \multicolumn{3}{|c|}{ Estimated mean (s.e.) } & \multicolumn{3}{|c|}{ ANCOVA } \\
\hline & Schizophrenia group & Bipolar disorder group & Control group & $F$ & $P$ & Post hoc \\
\hline 1 & $13.9(0.43)$ & $12.7(0.42)$ & $12.3(0.42)$ & 3.8 & 0.023 & $\begin{array}{l}\mathrm{SZ}>\mathrm{HC}(P=0.008) \\
\mathrm{SZ}>\mathrm{BD}(P=0.040)\end{array}$ \\
\hline 2 & $7.5(0.41)$ & $6.4(0.40)$ & $6.1(0.40)$ & 3.4 & 0.033 & $\mathrm{SZ}>\mathrm{HC}(P=0.012)$ \\
\hline 3 & $6.7(0.43)$ & $6.0(0.43)$ & $5.0(0.42)$ & 4.2 & 0.017 & $\mathrm{SZ}>\mathrm{HC}(P=0.005)$ \\
\hline
\end{tabular}

explain the graded effect of diagnosis. Therefore, only nominal $P$-values are presented.

\section{Cognitive, demographic and substance use factors}

Neither hand preference, education, IQ score, alcohol use, nonalcohol drug use nor smoking separately had a significant effect on the amplitude value in any of the components when group was also included in the regression model. The group effect and the graded response pattern (controls $<$ bipolar disorder $<$ schizophrenia) from the main analysis remained for all variables in all three components, except for component 3 where the group effect disappeared $(P=0.65)$ when education was included in the model.

\section{Clinical variables}

Lifetime episodes. The focus here was on history of psychosis in the bipolar disorder group, and history of depressive and manic episodes in the schizophrenia group. In bipolar disorder there was a significant effect of history of psychosis in component 3, whereby patients with psychosis showed a higher amplitude value than patients without such lifetime episodes $(t=2.2, P=0.028)$. In schizophrenia no difference was associated with a history of depression or mania. Additionally, in the bipolar disorder group, history of depression was associated with amplitude value in components $1 \quad(t=3.2, P=0.002)$ and $3(t=2.0, P=0.046)$, indicating differences in the mania-only subgroup $(n=9)$.

Current symptoms. In the bipolar disorder group, participants with elevated mood showed a lower amplitude value than those without elevated mood in components $3(t=-3.0, P=0.008)$ and $4(t=-2.1, P=0.038)$, whereas there was no effect of depressive or psychotic symptoms. In the schizophrenia group, no association with current symptom levels was found. 


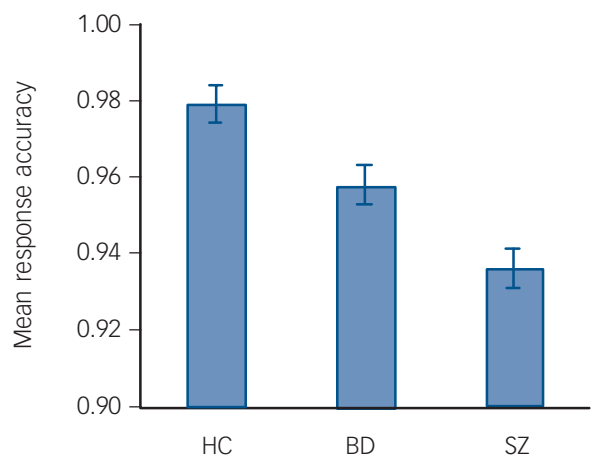

(b)

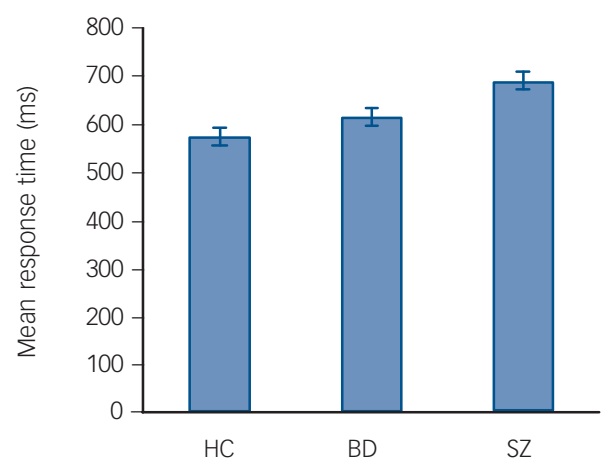

Fig. 1 Estimated means for response accuracy (a) and response time (b) in the working memory task for each group, after correction for gender and age. The error bars represent the standard error (BD, bipolar disorder group; HC, healthy control group; SZ, schizophrenia group).

Illness duration. There was no effect of illness duration on any of the components.

Medication. In bipolar disorder there was a significant effect of anxiolytics in component $4(t=2.1, P=0.043)$ but no effect of other medications. In schizophrenia there was an effect of anti-epileptics/lithium in component $1(t=2.3, P=0.022)$, but no effect of other medications.

Subgroups. There was no effect of bipolar disorder or schizophrenia spectrum subgroups on any of the components.

\section{Behavioural variables}

Neither accuracy nor response time alone had a significant effect on the amplitude value in any of the components when group was also included in the regression model. The group effect from the main analysis remained significant for both variables in all three components, except for accuracy in component 3. However, here the $P$-value for the group effect was just above the significance threshold $(P=0.055)$. The graded response pattern also remained in all components, except in component 1 when including response time, but this was probably affected by the exclusion of individuals with missing data (Table 2). The analysis of the effect of behavioural data on the amplitude values in each group separately showed that for accuracy there was no significant effect on the amplitude in any of the components in any of the groups. However, in the schizophrenia group there was a significant effect of response time on the amplitudes in all three components, where the amplitude values marginally decreased with response time (component $1 t=-2.6, P=0.013$; component $3 t=-3.6, P=0.001$; component $4 t=-2.1, P=0.043)$. However, it is worth noting that 15 people with schizophrenia were excluded from this analysis because of missing response time data (Table 2.)

\section{Discussion}

Our main finding was activation in the same brain networks for schizophrenia, bipolar disorder and control groups during a working memory task, but with a graded increased activation in key networks from controls to schizophrenia, with bipolar disorder in between. Nine independent components of brain activation related to the working memory task were identified, of which six were activations in brain regions previously found to be involved in working memory in healthy individuals. These included areas in the dorsolateral (Brodmann areas (BAs) 9/46) and ventrolateral (BAs 44/45/47) prefrontal cortex, premotor cortex (BAs 6/32) and parietal cortex (BAs 7/40) in both hemispheres. ${ }^{15,16}$ The last three components were deactivations in regions consistent with the main sites of the default mode network. $^{29}$

\section{Similar networks but different activation patterns}

Mainly the same brain areas were engaged in both patient and control groups, i.e. no disease-specific network or compensatory region was detected. This supports the notion that there are only subtle network abnormalities underlying cognitive impairment in psychotic disorders. However, in three of the task-positive components (1,3 and 4) there was a similar graded pattern of group differences in the amplitude of the fMRI signal, with participants in the bipolar disorder group showing stronger activation than the control group, and schizophrenia group participants showing stronger activation than both the control group and the bipolar disorder group. Considering the patients' young age, short duration of illness and low prevalence of symptoms at the time of the fMRI scanning, these results support the presence of qualitatively similar neurobiological abnormalities across disorders, with differences in severity between diagnoses. In addition, we used a large sample size, which further indicates that the fMRI results obtained are robust, and which enables additional analysis of subgroups. This is in contrast to the few other studies in the field; so far, there have been only two reported neuroimaging studies of working memory with a direct comparison between schizophrenia and bipolar disorder. ${ }^{13,14}$ The first study was based on 20 and 21 patients with schizophrenia and bipolar disorder respectively, compared with 38 controls. The working memory task was also different, with sets of abstract designs, and not an $n$-back task, most commonly used in fMRI studies of working memory. ${ }^{16}$ The other study was a pilot study using a 2-back task, performed by 10 patients with schizophrenia, 12 patients with bipolar disorder and 22 healthy participants. Both studies focused mainly on the prefrontal cortex, where decreased activation (hypoactivation) was found in the dorsolateral part in schizophrenia compared with controls, ${ }^{13}$ whereas increased activation (hyperactivation) was reported in the medial part in schizophrenia compared with both bipolar disorder and controls. ${ }^{14}$

\section{Hyperactivation}

Our results show that those in the patient groups had stronger activations than the control group. Several other neuroimaging studies of working memory have also shown hyperactivation in both bipolar disorder and schizophrenia, ${ }^{11,30}$ although not previously when compared with each other directly, except in the pilot study mentioned above. ${ }^{14}$ However, hypoactivation has also been a common finding. ${ }^{12}$ One explanation of these 


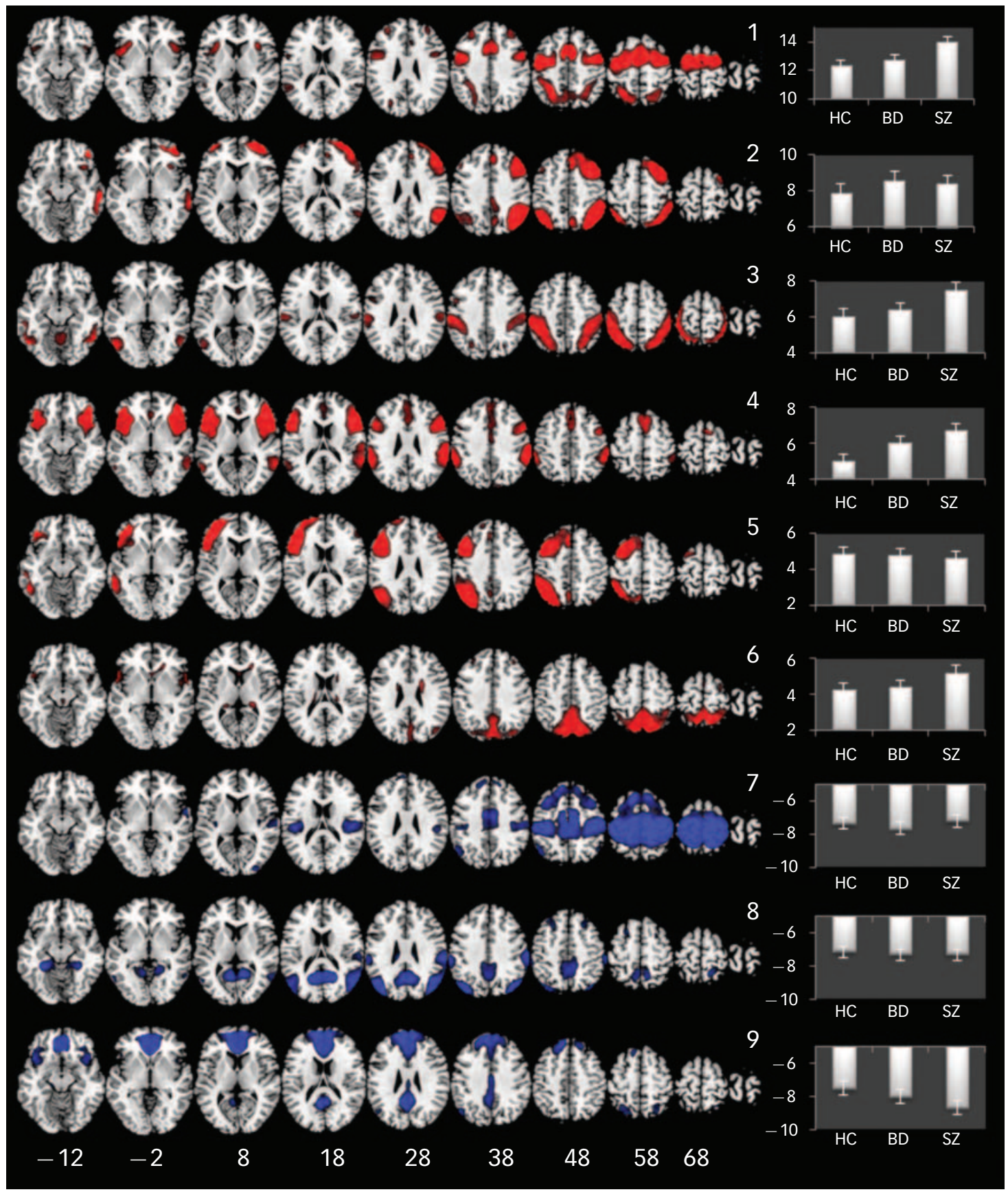

Fig. 2 Spatial maps of the nine independent components with the largest signal changes in the working memory task across all participants (left), and bar plots showing group differences in amplitude (right). Activations are shown in red and deactivations in blue. The bar plots show parameter estimates in arbitrary units (mean amplitude with standard error bars), corrected for gender and age. Significant group differences in amplitudes were found in components 1, 3 and 4 (BD, bipolar disorder; HC, healthy controls; SZ, schizophrenia).

inconsistent findings has been that the brain activation of people with schizophrenia and healthy controls may be represented by different, but overlapping, inverted U-curve shapes of activation. ${ }^{31,32}$ According to such a model, patients will show stronger activation than controls even if task difficulty is low. This fits well with the behavioural data from our study, which showed that both patient groups had a high performance level, and it suggests that the patients had to use more cognitive resources than the controls to perform the same task. The current results further suggest that the activation pattern in bipolar disorder fits into the same model, with a less abnormal inverted U-curve of activation compared with schizophrenia. However, the results may also partly reflect the graded pattern of task performance measures, with the bipolar disorder group results lying between the two other groups, even if the secondary analyses of task performance effects did not show any strong influence on the main results. Altogether, the grading of activation levels from controls to bipolar disorder to schizophrenia may reflect both the increased difficulty in task performance across groups and the compensatory increased recruitment of brain networks in patients.

\section{Working memory network}

The three components that showed group differences were all in frontal and parietal brain regions consistent with the well-known working memory network. Previous neuroimaging studies, most 
of them in schizophrenia, have focused on and found abnormalities not only in the prefrontal cortex in particular (cf. the discussion about hyper- and hypoactivation) but also in the parietal cortex. ${ }^{12,33}$ It has been proposed that structural and functional alterations in schizophrenia might start in the parietal lobe and progress to frontal regions. ${ }^{33}$ One could speculate that the same applies to bipolar disorder, but with a lower magnitude. Further, the fact that we found group differences in components that involved all the typical working memory areas supports the notion that both schizophrenia and bipolar disorder involve abnormalities in a distributed network of brain regions, not only in single regions. ${ }^{12}$

\section{Association with clinical variables}

Secondary analyses were performed to investigate whether the finding of a graded response pattern across diagnoses could be explained by factors other than diagnostic group. The results showed that certain clinical variables were associated with timecourse amplitudes. Most of the variation in amplitude values was found in bipolar disorder, where particularly component 3 , involving parietal brain regions, seemed to be associated with clinical factors. However, the effect size was small for all variables. Of special interest, there was a significant effect of history of psychosis in component 3. Here, the bipolar disorder group patients with psychosis showed an amplitude response pattern that more closely resembled that of the schizophrenia group than that of the bipolar disorder group patients without such lifetime episodes. Thus, history of psychosis seemed to explain some of the graded response pattern in parietal regions. In bipolar disorder there was also an association between history of depression and amplitude value in two of the components, indicating differences in the mania-only subgroup $(n=9)$. Further, two components were associated with current elevated mood in bipolar disorder. Here, patients with elevated mood symptoms showed a response pattern that was more similar to that of the control group. In schizophrenia, neither lifetime episodes nor current symptoms seemed to affect the component time-course amplitudes. Regarding the impact of medication, there were few effects on amplitude value within each patient group. The only associations were found for anxiolytics in one component in bipolar disorder, and for anti-epileptics/lithium in one component in schizophrenia. This is in line with two reviews showing few general effects of medication on functional neuroimaging findings in schizophrenia and bipolar disorder. ${ }^{34,35}$ Except for the effects of these clinical characteristics, there were few variables that seemed to affect the main results. Altogether, the results of the secondary analyses indicate that the graded activation pattern across diagnostic groups is a robust phenomenon, which is in line with a continuum of neuronal abnormalities across psychotic disorders.

\section{Limitations}

Some limitations of the study should be acknowledged. First, the participant groups were not matched on variables such as gender, age, education, IQ score, substance use and task performance. The main reason for this was that we chose to include a large sample with the possibility of looking at subgroups, rather than a smaller sample with matched groups on a few key variables. However, since patient investigations are always confounded because of heterogeneous diagnoses and a number of cognitive, clinical and behavioural factors that may influence the results, it is important to know the effect of the variables that differ between groups and interpret the results in light of these effects. The alternative of including covariates in the main analysis increases the risk of correcting for variables that are related to the illness (e.g. IQ score, drug use or symptoms). Therefore, we only corrected for variables that were significantly different between groups, but which were not directly linked to the illness (gender and age). Further, we tested the effect of several other factors that might explain the main results. Second, we only found significant differences in fMRI signal amplitudes between the schizophrenia and control groups, and not between the schizophrenia and bipolar disorder groups (except for one component) or between the bipolar disorder and control groups. This could partly be due to heterogeneity in the bipolar disorder group, and the fact that schizophrenia is a more severe disorder. However, the main point of interest was the graded response pattern that was present in all the components with group effects. Third, we did not measure the effect on the data of phase of menstrual cycle, which has been found to affect working memory function. ${ }^{36}$ Fourth, there were missing data for some variables, especially on the behavioural measures $(10 \%)$ due to technical problems.

\section{Implications}

These findings add a new level of explanation to the overlapping clinical characteristics and genetic factors in schizophrenia and bipolar disorder. ${ }^{1,2,4}$ The results suggest that the two disorders should be perceived as dimensionally different, or different in the degree to which specific substrates or neural mechanisms are perturbed, rather than categorically separate entities. It could be speculated that the findings support the concept of a psychosis continuum. ${ }^{37-40}$ However, more research is needed before the neurobiological substrates can be implemented in future revisions of DSM. ${ }^{37-39}$

\section{Funding}

The study was financially supported by the Research Council of Norway (grant numbers 167153/V50, 163070/V50 and 183782/V50) and the South-Eastern (2004-123) and Western (91141) Norway Regional Health Authority.

\section{Acknowledgements}

The authors thank the study participants for their contribution, and the clinicians who were involved in patient recruitment and clinical assessments. Special thanks are due to Anne Hilde Farstad and the staff at the Department of Radiology and Nuclear Medicine, Oslo University Hospital, and Eivind Bakken of the Thematically Organised Psychosis study, for providing technical assistance.

Christine Lycke Brandt, MSC, K.G. Jebsen Centre for Psychosis Research, Institute of Clinical Medicine, University of Oslo, and Division of Mental Health and Addiction, Oslo University Hospital, Oslo; Tom Eichele, MD, PhD, Department of Biological and Medical Psychology, University of Bergen, Bergen; Ingrid Melle, MD, PhD, K.G. Jebsen Centre for Psychosis Research, Institute of Clinical Medicine, University of Oslo, and Division of Mental Health and Addiction, Oslo University Hospital, Oslo; Kjetil Sundet, PhD, Department of Psychology, University of Oslo, K.G. Jebsen Centre for Psychosis Research, Institute of Clinical Medicine, University of Oslo, and Division of Mental Health and Addiction, Oslo University Hospital, Oslo; Andrés Server, MD, Section of Neuroradiology, Department of Radiology and Nuclear Medicine, Oslo University Hospital, Oslo; Ingrid Agartz, MD, PhD, K.G. Jebsen Centre for Psychosis Research, Institute of Clinical Medicine, University of Oslo, and Department of Psychiatric Research, Diakonhjemmet Hospital, Oslo, Oslo, and Department of Psychiatric Research, Diakonhjemmet Hospital, Oslo,
Norway, and Department of Clinical Neuroscience, Psychiatry Section, Karolinska Institute, Stockholm, Sweden; Kenneth Hugdahl, PhD, Department of Biological and Medical Psychology, University of Bergen, and Division of Psychiatry, Department of Radiology, Haukeland University Hospital, Bergen, Norway; Jimmy Jensen, PhD, Centre for Psychology, Kristianstad University, Kristianstad, Sweden, K.G. Jebsen Centre for Psychosis Research, Institute of Clinical Medicine, University of Oslo and Division of Mental Health and Addiction, Oslo University Hospital, Oslo, Norway; Ole A. Andreassen, MD, PhD, K.G. Jebsen Centre for Psychosis Research, Institute of Clinical Medicine, University of Oslo, and Division of Mental Health and Addiction, Oslo University Hospital, Oslo, Norway

Correspondence: Christine Lycke Brandt, Division of Mental Health and Addiction, Psychosis Research Unit/TOP, Ullevål Hospital, Building 49, PO Box 4956 Nydalen, N-0424 Oslo, Norway. Email: c.l.brandt@medisin.uio.no

First received 18 Mar 2013, final revision 5 Jul 2013, accepted 25 Oct 2013 


\section{References}

1 Craddock N, O'Donovan MC, Owen MJ. Psychosis genetics: modeling the relationship between schizophrenia, bipolar disorder, and mixed (or 'schizoaffective') psychoses. Schizophr Bull 2009; 35: 482-90.

2 Lichtenstein P, Yip BH, Bjork C, Pawitan Y, Cannon TD, Sullivan PF, et al. Common genetic determinants of schizophrenia and bipolar disorder in Swedish families: a population-based study. Lancet 2009; 373: 234-9.

3 Purcell SM, Wray NR, Stone JL, Visscher PM, O'Donovan MC, Sullivan PF, et al. Common polygenic variation contributes to risk of schizophrenia and bipolar disorder. Nature 2009; 460: 748-52.

4 Van Snellenberg JX, de Candia T. Meta-analytic evidence for familial coaggregation of schizophrenia and bipolar disorder. Arch Gen Psychiatry 2009; 66: 748-55

5 Green MF. Cognitive impairment and functional outcome in schizophrenia and bipolar disorder. J Clin Psychiatry 2006; 67 (suppl 9): 3-8; discussion 36-42.

6 Simonsen C, Sundet K, Vaskinn A, Birkenaes AB, Engh JA, Færden A, et al Neurocognitive dysfunction in bipolar and schizophrenia spectrum disorders depends on history of psychosis rather than diagnostic group. Schizophr Bull 2011; 37: 73-83.

7 McIntosh AM, Whalley HC, McKirdy J, Hall J, Sussmann JE, Shankar P, et al. Prefrontal function and activation in bipolar disorder and schizophrenia. Am J Psychiatry 2008; 165: 378-84.

8 Whalley HC, Papmeyer M, Sprooten E, Lawrie SM, Sussmann JE, McIntosh AM. Review of functional magnetic resonance imaging studies comparing bipolar disorder and schizophrenia. Bipolar Disord 2012; 14: 411-31.

9 Calhoun VD, Sui J, Kiehl K, Turner J, Allen E, Pearlson G. Exploring the psychosis functional connectome: aberrant intrinsic networks in schizophrenia and bipolar disorder. Front Psychiatry 2011; 2: 75

10 Hall J, Whalley HC, Marwick K, McKirdy J, Sussmann J, Romaniuk L, et al. Hippocampal function in schizophrenia and bipolar disorder. Psychol Med 2010; 40: 761-70.

11 Adler CM, Holland SK, Schmithorst V, Tuchfarber MJ, Strakowski SM. Changes in neuronal activation in patients with bipolar disorder during performance of a working memory task. Bipolar Disord 2004; 6: 540-9.

12 Glahn DC, Ragland JD, Abramoff A, Barrett J, Laird AR, Bearden CE, et al. Beyond hypofrontality: a quantitative meta-analysis of functional neuroimaging studies of working memory in schizophrenia. Hum Brain Mapp 2005; 25: 60-9.

13 Hamilton LS, Altshuler LL, Townsend J, Bookheimer SY, Phillips OR, Fischer J, et al. Alterations in functional activation in euthymic bipolar disorder and schizophrenia during a working memory task. Hum Brain Mapp 2009; 30: 3958-69.

14 Milanovic SM, Thermenos HW, Goldstein JM, Brown A, Gabrieli SW, Makris N, et al. Medial prefrontal cortical activation during working memory differentiates schizophrenia and bipolar psychotic patients: a pilot FMRI study. Schizophr Res 2011; 129: 208-10.

15 Cabeza R, Nyberg L. Imaging cognition II: an empirical review of 275 PET and fMRI studies. J Cogn Neurosci 2000; 12: 1-47.

16 Owen AM, McMillan KM, Laird AR, Bullmore E. N-back working memory paradigm: a meta-analysis of normative functional neuroimaging. Hum Brain Mapp 2005; 25: 46-59.

17 Schneider F, Habel U, Reske M, Kellermann T, Stocker T, Shah NJ, et al. Neural correlates of working memory dysfunction in first-episode schizophrenia patients: an fMRI multi-center study. Schizophr Res 2007; 89: $198-210$.

18 Moller HJ. Bipolar disorder and schizophrenia: distinct illnesses or a continuum? J Clin Psychiatry 2003; 64 (suppl 6): 23-7; discussion 8.
19 American Psychiatric Association. Diagnostic and Statistical Manual of Mental Disorders (4th edn) (DSM-IV). APA, 1994.

20 Spitzer RL, Williams JB, Kroenke K, Linzer M, deGruy FV, Hahn SR, et al. Utility of a new procedure for diagnosing mental disorders in primary care. The PRIME-MD 1000 study. JAMA 1994; 272: 1749-56.

21 First MB, Spitzer RL, Gibbon M, Williams JBW. Structured Clinical Interview for DSM-IV Axis I Disorders: Patient Edition (SCID-P), Version 2. Biometrics Research, 1995.

22 Ringen PA, Lagerberg TV, Birkenaes AB, Engn J, Faerden A, Jonsdottir $\mathrm{H}$, et al. Differences in prevalence and patterns of substance use in schizophrenia and bipolar disorder. Psychol Med 2008; 38: 1241-9.

23 Aminoff SR, Jensen J, Lagerberg TV, Andreassen OA, Melle I. Decreased self-reported arousal in schizophrenia during aversive picture viewing compared to bipolar disorder and healthy controls. Psychiatry Res 2011 185: 309-14.

24 Hugdahl K, Rund BR, Lund A, Asbjornsen A, Egeland J, Ersland L, et al. Brain activation measured with fMRI during a mental arithmetic task in schizophrenia and major depression. Am J Psychiatry 2004; 161: 286-93.

25 Haatveit BC, Sundet K, Hugdahl K, Ueland T, Melle I, Andreassen OA. The validity of $d$ prime as a working memory index: results from the 'Bergen n-back task'. J Clin Exp Neuropsychol 2010; 32: 871-80.

26 Himberg J, Hyvarinen A, Esposito F. Validating the independent components of neuroimaging time series via clustering and visualization. Neuroimage 2004; 22: 1214-22.

27 Bell AJ, Sejnowski TJ. An information-maximization approach to blind separation and blind deconvolution. Neural Comput 1995; 7: 1129-59.

28 Cole DM, Smith SM, Beckmann CF. Advances and pitfalls in the analysis and interpretation of resting-state FMRI data. Front Syst Neurosci 2010; 4: 8.

29 Raichle ME, MacLeod AM, Snyder AZ, Powers WJ, Gusnard DA, Shulman GL. A default mode of brain function. Proc Natl Acad Sci USA 2001; 98: 676-82.

30 Callicott JH, Bertolino A, Mattay VS, Langheim FJ, Duyn J, Coppola R, et al. Physiological dysfunction of the dorsolateral prefrontal cortex in schizophrenia revisited. Cereb Cortex 2000; 10: 1078-92.

31 Karlsgodt KH, Sanz J, van Erp TG, Bearden CE, Nuechterlein KH, Cannon TD. Re-evaluating dorsolateral prefrontal cortex activation during working memory in schizophrenia. Schizophr Res 2009; 108: 143-50.

32 Callicott JH, Mattay VS, Verchinski BA, Marenco S, Egan MF, Weinberger DR. Complexity of prefrontal cortical dysfunction in schizophrenia: more than up or down. Am J Psychiatry 2003; 160: 2209-15.

33 Yildiz M, Borgwardt SJ, Berger GE. Parietal lobes in schizophrenia: do they matter? Schizophr Res Treatment 2011; 2011: 581686.

34 Roder $\mathrm{CH}$, Hoogendam JM, van der Veen FM. FMRI, antipsychotics and schizophrenia. Influence of different antipsychotics on BOLD-signal. Curr Pharm Des 2010; 16: 2012-25.

35 Hafeman DM, Chang KD, Garrett AS, Sanders EM, Phillips ML. Effects of medication on neuroimaging findings in bipolar disorder: an updated review. Bipolar Disord 2012; 14: 375-410.

36 Jacobs E, D'Esposito M. Estrogen shapes dopamine-dependent cognitive processes: implications for women's health. J Neurosci 2011; 31: 5286-93.

37 Fischer BA, Carpenter WT. Will the Kraepelinian dichotomy survive DSM-V? Neuropsychopharmacology 2009; 34: 2081-7.

38 Moller HJ. Is the overlap of neurobiological and psychopathological parameters large enough to give up the dichotomic classification? Psychiatr Ann 2010; 40: 163-7.

39 Vieta E, Phillips ML. Deconstructing bipolar disorder: a critical review of its diagnostic validity and a proposal for DSM-V and ICD-11. Schizophr Bull 2007; 33: 886-92.

40 Editorial. A continuum of psychosis? Lancet 1987; 2: 889-90. 